\title{
Evaluation of Supra-Orbital Approach through Eyebrow Incision for Treating Tumors in Anterior Cranial Fossa and Supra-sellar Region
}

A.K.Ali ${ }^{1}$,W.A.Badawy, M.M.Adawy, M.A.El awady and B.M.Mahmoud

Neurosurgery,Dept., Faculty of Medicine, Benha Univ., Benha, Egypt

E-Mail:Baher56@gmail.com

\begin{abstract}
This technique is suitable to lesions situated in the region of the anterior fossa, suprasellar cisterns, parasellar region and Sylvian fissure. Should assess the supra-orbital approach through eyebrow entry point to surgical extraction from claiming tumors to foremost cranial fossa What's more supra-sellar area. A $50 \mathrm{~mm}$ entry point in the eyebrow What's more a supraorbital minicraniotomy will be performed. 20 patients harboring separate lesions were worked around for beneficial postoperative Furthermore cosmea outcomes. We infer that thisapproach is safe Furthermore advantageous in chose cases. 20 patients harboringdifferent lesions were worked looking into with useful postoperative Furthermore cosmea effects. We infer that thisapproach will be protected What's more advantageous in chose cases.
\end{abstract}

KeyWords: Anterior skull base, Eyebrow incision, Minimally invasive surgicalapproach, Sellar Region.

\section{Introduction}

Those supraorbital eyebrow craniotomy will be a anterolateral approach that permits those specialist should location different pathology of the foremost cranial fossa, parasellarregion, proximalsylvian fissure, ipsilateral Also contralateral locales of the circis siliquastrum of Willis, basal frontal lobe, Furthermore ventral brainstem. When supplemented for intracranial endoscopy, lesions of the parallel deep set sinus, pituitary fossa, contralateral circis siliquastrum about Willis, Also ipsilateralretroorbital space might be tended to. Those supraorbital methodology gives those A large portion regulate surgical trajectory of the frontal pole Also a few mesial Furthermore orbital parts of the frontal projection (with no require for altered cerebrum retraction) and also should orbitofrontal and skull base lesions Furthermore the individuals in the mesial transient flap [1].

Those possibility points of interest of the minimally obtrusive eyebrow craniotomy are identified with shorter surgical methods What's more healing facility stays, lesquerella agent trauma, enhanced easing starting with pain, Also better cosmea results. The effortlessness Also flexible of the eyebrow supraorbital methodology make it a standout amongst the The majority productive skull build strategies [2].

Normal difficulties of the supraorbital eyebrow craniotomy are transient brow deadness from withdrawal damage of the supraorbital nerve. In spite of the fact that An normal protestation in the right on time postoperative period, this manifestation might have been infrequently lasting. Also, frontalis shortcoming in the punctual postoperative stage , presumably from extending of the frontalis extension of the facial nerve. This might have been also transitory in the dominant part of cases[3].

Reason. Should assess those supra-orbital approach through eyebrow entry point to surgical extraction of tumors done foremost cranial fossa What's more suprasellar locale.

\section{Patients and methods}

This review examine might have been carried out for 20 patients for separate obsessive lesions for foremost skull base and sellar districts Furthermore subjected will surgery Toward frontolateral craniotomy by means of eyebrow. skin incision.

\section{Inclusion criteria}

- Patients with tumors of the anterior cranial base and sellar region, with no privilege for age or gender.

- Lesions were considered indicated for surgery if the lesion produced mass effect, hormonal disturbance indicating surgery or a progressive disorder needing early intervention and not only follow up.

\section{Exclusion criteria}

Lesions which need more tailored approaches with:

- Extension to the lateral middle cranial fossa, posterior fossa, nasal sinuses.

- Extensive infiltration of neurovascular structures.

\subsection{Methodology}

All patients involved in this study are managed according to the following scheme:

I. Clinical assessment: history, general examination, and neurological examination.

II. Investigations

1. Routine laboratory investigations

2. Hormonal assays in case of suspected endocrinal abnormalities.

3. Radiological: CT and/or MRI study of brain with and without contrast were done for every patient preoperatively.

4. Histopathological examination of all specimens by neuropathologist.

III. Treatment:

\section{A- Surgical treatment}

Surgical treatment was done in all patients included in this study in the form of frontolateral craniotomy via superciliary skin incision.

\section{1- Position}

The patient was placed in a supine position with the head fixed in a Mayfield head holder. The head is rotated $10^{\circ}-60^{\circ}$ to the contralateral side, according to location of the lesion. 


\section{Incision}

Eye forehead skin entry point from Exactly parallel of the supraorbital score of the parallel edge of the eye brow, then 2or3 sutures were made in the subcutaneous tissue Furthermore retracting the skin rearward and suturing it of the drapes. Those skin over those frontal range might be effortlessly withdrew and assembled with addition subcutaneous analyzation. Throughout dissection, introduction of the frontal Furthermore orbital muscles Also its withdrawal descending towards those globe ought to make confined to a essential minimum, should keep away from postoperative periorbital edema.

\section{3- Approach}

18 Patients were worked from good side, 2 situations starting with left side. The point when craniotome might have been accessible just those keyhole might have been completed et cetera rectangular craniotomy $2.5 \times 3 \mathrm{~cm}$ might have been carried out Toward craniotome. The point when there might have been no craniotome, An little drill might have been used to settle on 4 burr holes; person keyhole, particular case 2. $5 \mathrm{~cm}$ behind (temporal), anterior frontal $(3 \mathrm{~cm}$ average of the keyhole), posterior frontal $(2.5 \mathrm{~cm}$ behind foremost frontal) emulated by extending those burr gaps starting with their undersurface without really expanding external breadth. This permitted simple section of the aide Furthermore Gigli saw. Afterward the dura is opened done a C-shaped style based towards those base, for addition CSF waste Eventually Tom's perusing opening of the sylvian crevice. At that point microsurgical procedure might have been used to handle those lese greatness. During the wind of operation those dura will be shut in An water tight fashion, those bone fold may be put those burr gaps were fixed by bone dust, those periosteum may be shut should stay with those bone fold set up then those galea is shut Eventually Tom's perusing intruded on absorbable sutures and the skin will be shut in a subcuticular cosmea style again a little suction curettage channel.

\section{4- Excision}

Total excision was the target in all cases, but this was not possible in some cases due to infiltration, encasing or attachment to vital structures.The excision was graded total, subtotal ( $\geq 70 \%$ was removed) and partial ( $<70 \%$ was removed).

\section{5- Adjuvant surgical procedures}

\section{Results}

The data collected from the 20 cases that were operated upon during this work were studied, and the results were concluded, compared and evaluated.

Gender: The study included 10 males and 10 females operated upon by the frontolateral craniotomy via eyebrow skin incision. e.g. CSF shunting, when necessary (which is not needed in our study).

\section{B- Adjuvant Therapy}

Radiosurgery, radiotherapy and chemotherapy was done according to histopathology.

\section{C- Medical treatment}

- Antiepileptic drugs.

- Patients with endocrinopathies were referred to endocrinologist.

- Symptomatic treatment.

\section{IV. follow up}

All patients were assessed clinically and radiologicallyimmediately post -operative and 3 months after surgery to evaluate:-

1. Approach related complications.

2. Approach related difficulties.

3. Extent of lesions resection.

4. Post- operative scar.

\section{Outcome}

Was estimated according to the following scale (Odom's criteria for evaluation of outcome):

\section{A. Excellent}

- Improvement of pre-operative neurological deficit.

- No additional deficits.

- The patient is doing his activities well.

\section{B. Good}

- Improvement of pre-operative neurological deficits, with persistence of minimal symptoms or additional deficits that is easily treated.

- The patient is doing his activities well.

- C. Poor:

- No-Improvement of pre-operative neurological deficits.

- Additional post-operative deficits or untreatable complications.

\section{Death}

- Operative mortality was considered in patients who died within one month after the operation.

- Complications that could be managed adequately and effectively, as diabetes insipidus, hydrocephalus, medically controllable endocriopathies, temporary deficits that resolved adequately were not considered as bad prognostic factors.

- Craniotomy related complications, as scars, temporal muscle wasting, and skull defects, were considered as bad prognostic factors if found to cause unpleasant cosmetic effects to the patient Age: The youngest patient operated upon by the frontolateral craniotomy via eyebrow skin incision during this study was 11 years while the oldest was 60 years and the mean age was 35.5

\section{Symptoms}

Shortest duration of symptom was 1 month $(2 / 20)$ and the longest duration was 36 months (1/20) Table 


\section{(1), Fig (1)}

The most common presenting symptom was diminution of vision in $90 \%$ (18/20) of cases followed by headache in $80 \%(16 / 20)$ Table (2).

\section{SIGNS}

The most common clinical sign in this study was affection of visual acuity in $90 \%$ of cases followed by field defects in $80 \%$ of cases and abnormal fundus examination in $50 \%$ of cases Table (3), Fig (2).

\section{Radiological analysis \\ Size and nature}

The smallest lesion operated upon in this study was $2 \mathrm{~cm}$ while the largest was $6 \mathrm{~cm}$; lesions had an average size was $3.15 \mathrm{~cm}$ Table (5), Fig (3). It should be noted that the size was given to the longest sagittal diameter of the lesion. The radiological appearance was purely solid lesion in 17 patients (85\%), mixed solid and cystic lesions in 3 patients (15\%), and none were purely cystic Table (6).

Site and extension As regard the location and extension of the lesion; all the 20 lesions were around the sella with variable degrees of extension ( 8 pituitary adenomas, 3 craniopharyngiomas, and 9 meningiomas) Table (7).

\section{Enhancement}

Post-contrast studies (CT and/or MRI) showed homogenous enhancement in 13 patients (65\%), heterogeneous enhancement in 7 patients (35\%) Table (8).

\section{Hydrocephalus and surrounding oedema}

Preoperative hydrocephalus was present in one patient (5\%). Surrounding oedema was present in 4 patients (20\%).

\section{Surgical finding}

\section{The selected approach}

Among 20 patients; 18 patients $(90 \%)$ were operated via the right frontolateral approach, and 2 patients $(10 \%)$ via the left frontolateral approach.

\section{Nature of the lesion}

The lesion was found to be firm and tough in 9 patients (45\%), soft and suckable in 8 patients (40\%), mixed cystic and solid in 3 patients (15) \%.

\section{Attachment of the lesion to the surroundings Extent of surgical excision Comment}

The lesions which were totally removed in 9 cases: 6 meningiomas , 1 craniopharangioma and 2 adenomas.

The lesions which were subtotally removed in $\mathbf{7}$ cases: 3 meningiomas 1 craniopharyngioma and 4 pituitary adenomas.

The lesions which were partially resected in $\mathbf{4}$ cases: 2 pitutary adenoma and 1 craniopharyngioma.

\section{Postoperative medical treatment}

All patients were given antibiotics for at least first postoperative week. Antiepileptic drugs were given to all patients intraoperative and continued for at least first 3 months post-operative. Endocrinal abnormalities were referred to endocrinologist to be properly managed.

Adjuvant therapy: - It was in the form of

1. Radiotherapy in 2 patients; atypical meningioma.

2. Chemotherapy in 2 patients; the same cases of radiotherapy.

3. Radiosurgery (Gamma knife):- in 4 patients; 2 meningiomas, 1 craniopharyngiomas and 1 pituitary adenoma.

\section{Histopathological findings}

Different pathological lesions were treated: There were 9 meningiomas, 3 craniopharyngiomas, 8 pituitary adenomas Table (11).

\section{Hospital stay \\ Follow up findings}

All patients were assessed clinically and radiologicallyimmediately post-operative and all patients were followed up postoperatively clinically, radiological 3 months, 6 months and 1 year after surgery to evaluate:-

- Extent of lesions resection.

- Approach related difficulties.

- Approach related complications.

- Post- operative scar.

\section{Clinical follow up}

Postoperative visual evaluation showed that in 16 patients of 20 patients (90\%) with visual problems the vision improved, in 4 patients it was the same as preoperative and not improved with follow up, and the vision did not deteriorate in any patient.

Postoperative endocrinal evaluation showed improvement in 1 patient of pituitary adenoma in which menstruation became regular and prolactin level was normalized. 3 patients developed temporary diabetes insipidus.

Two patients presented by fits; all of them were controlled by antiepileptic drugs preoperative and remain free of fits during the period of follow up.

Specific parameters related to the approach (local complication).

Transient Periorbital oedema occurs in all patients and maximally resolves by the end of first week.

Supraorbital hypoesthesia was evident in 16 patients (80\%), 13 patients of them were temporary and resolved within few months and was permanent in 3 patients.

Wound infection; during follow up period 1 patient showed superficial wound infection which subsides under antibiotic therapy. 
Cosmetic problems; occur in 1 patients $(5 \%)$ in which patient had visible scar.

Temporalis muscle wasting or pain; was not found in any patient.

Eye brow elevation was lost early in 15 patients, during follow up it subsided in 14 patients, remained in 1 patient.

\section{Comment}

The patients who have excellent outcome were:-

\section{Meningiomas}

1 Craniopharyngiomas with postoperative improvement of vision.

3 Pituitary adenomas; one patient had prolactinoma with postoperative improvement of endocrinal manifestations and normalization of prolactin level with medical treatment. The other one had postoperative improvement of vision.

The patients who had good outcome were:-

1 Craniopharyngioma in which vision improved but hormonal disturbance still need continuous replacement therapy during follow up period.

5 Pituitary adenomas in which vision improved but hormonal disturbance still need continuous replacement therapy during follow up period.

1 Meningioma in which headache and dizziness improved but visual acuity did not improve.

The patients who had Poor outcome were:-

One patient with Craniopharyngioma this patient in spite of mild improvement of some preoperative symptoms but he had unsatisfactory cosmoses in the form of visible scar and persistence of other preoperative symptoms.

Cosmetic outcome and patient satisfaction:

Since the incision of the frontolateral approach in this study is placed in the eyebrow, it has a more potential of producing a less visible or disfiguring scar but this was noticed in one patient had a visible scar.

The craniotomy, weather visible or not is also a determining cosmetic factor. In this study patients were satisfied from this cosmetic point of view.

Another point of importance is eyebrow elevation. In 15 patients operated upon in this study eyebrow elevation is temporary lost and tends to recover by time except in one patient in which it remains weak. This is due to striping of the frontals muscle from its origin, thus it is important during closure of the wound to reapply the pericranium and to suture the muscle at its appropriate layer. In some case due to adhesions between the pericranium and muscle, the mobility becomes limited and restricted.

\section{Illustrative cases}

Case (1)

22 years old, female complaining of 2 months history of progressive visual deterioration

Fully conscious, full motor power light

Rt. Eye, diminished visual acuity, perception of

Lt. eye, counting fingers, 4 meters

MRI with contrast showed a suprasellar mass likely a tuberculumsellae meningioma

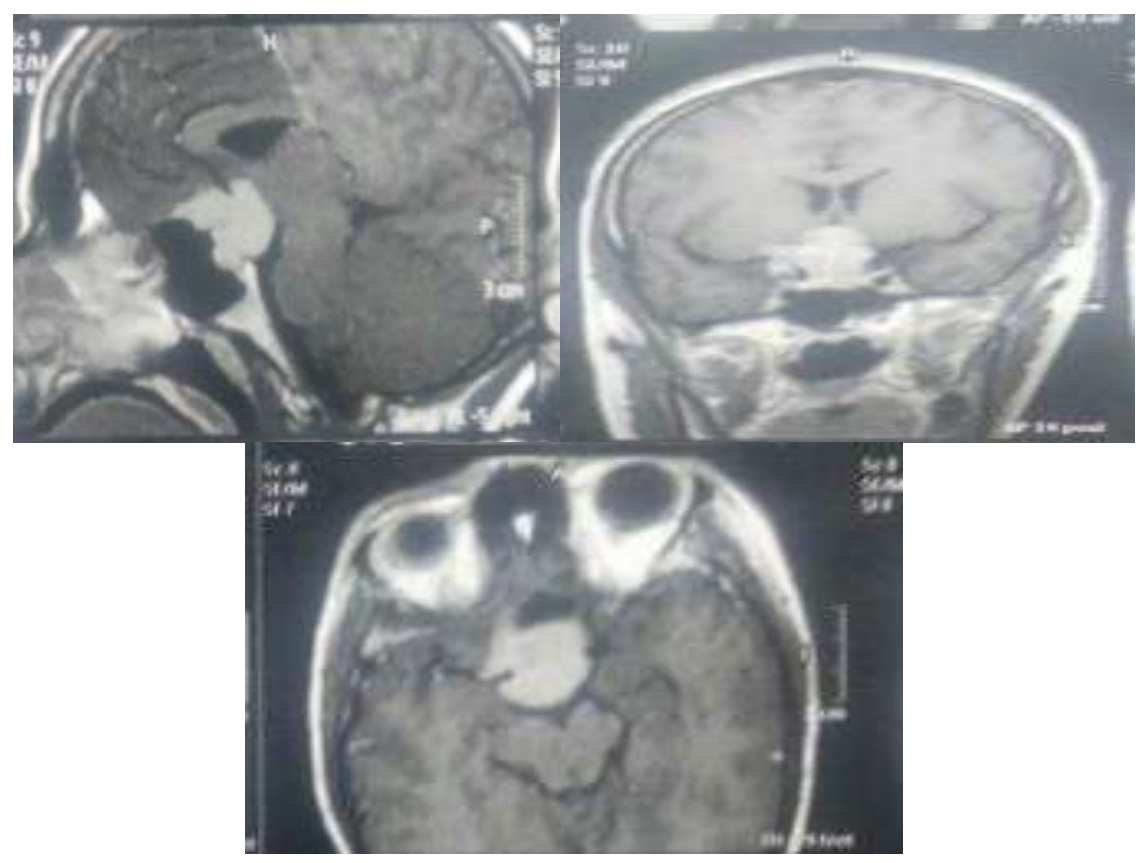

Case 1, (preoperative MRI with contrast, sagittal, coronal\& axial cuts showed an inhanced mass at suprasellar region likely a tuberculumsellae meningioma)

Post operative patient was fully conscious, full motor power with mild headache, vomiting and hyperglycemia which was controlled medically with good total tumor 


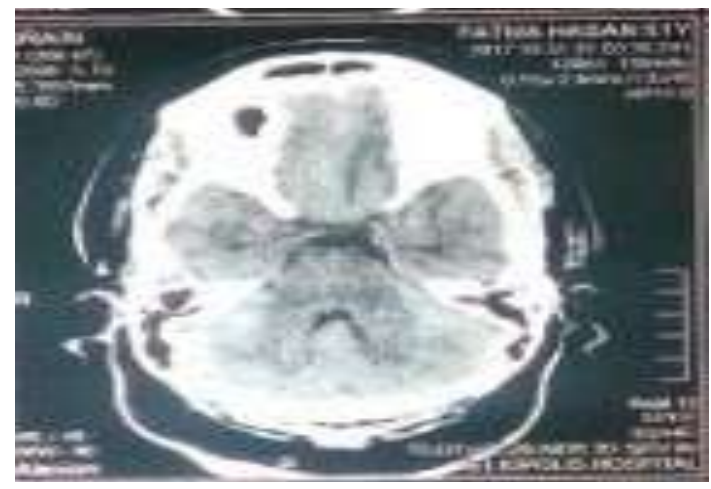

Case (1) postoperative ct. axial cut shows total excision of the mass with clear tumor bed

Case (2)

A 47 years old male patient, complaining of gradual diminution of vision and intermittent headaches with easy fatigability and polyurea .

Visual field showed bitemporal henianopsia with diminished visual acuity

Rt. Eye, counting fingers, 4 meters.

Lt. Eye, counting fingers, 2 meters.

Fully conscious and full motor power.

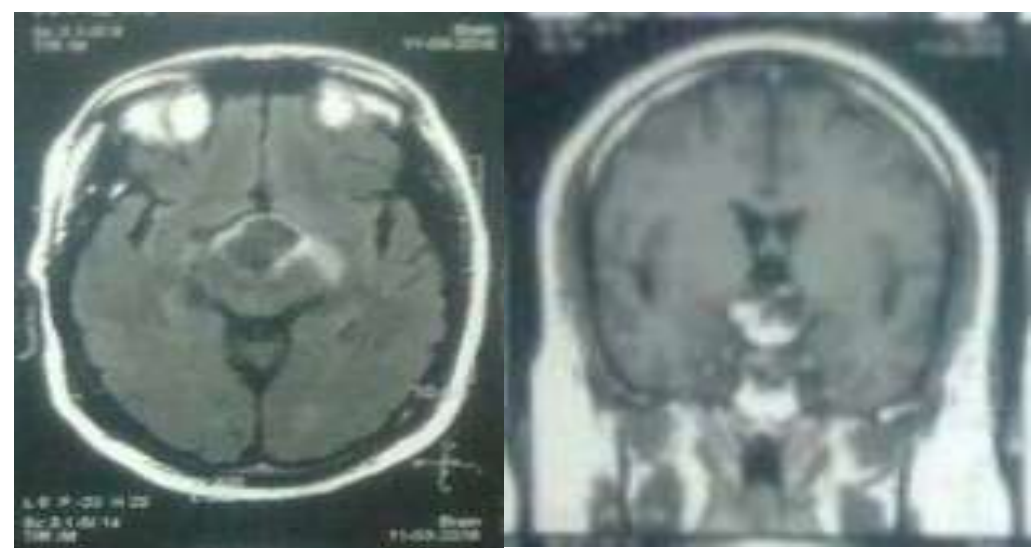

Case(2) Preoperative MRI with contrast, axial and coronal cuts showed a sellar and suprasellar mass which is partially enhanced with cystic component

Postoperative he was fully conscious with full motor power, visual acuity was the same as preop.

Urine output was $170 \mathrm{ml}$ per hour with s. sodium $139 \&$ s. potassium, 3.9 in first day.

Pathology was cystic craniopharyngioma WHO grade I .

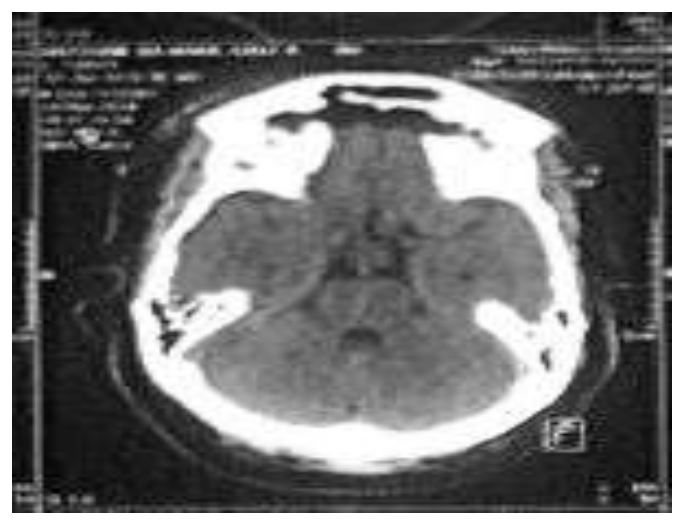

Case (2) postoperative ct. , axial cut shows total excision of the mass with clear tumor bed 
Table (1) Duration of symptoms in months.

\begin{tabular}{lcc}
\hline Duration of symptoms & Frequency & Percent \\
\hline 1 - 6 months & 7 & 35 \\
$\mathbf{7}$-12 months & 4 & 20 \\
13 -18 months & 5 & 25 \\
19 -24 months & 3 & 15 \\
More than 24 months & 1 & 5 \\
Total & 20 & 100 \\
\hline
\end{tabular}

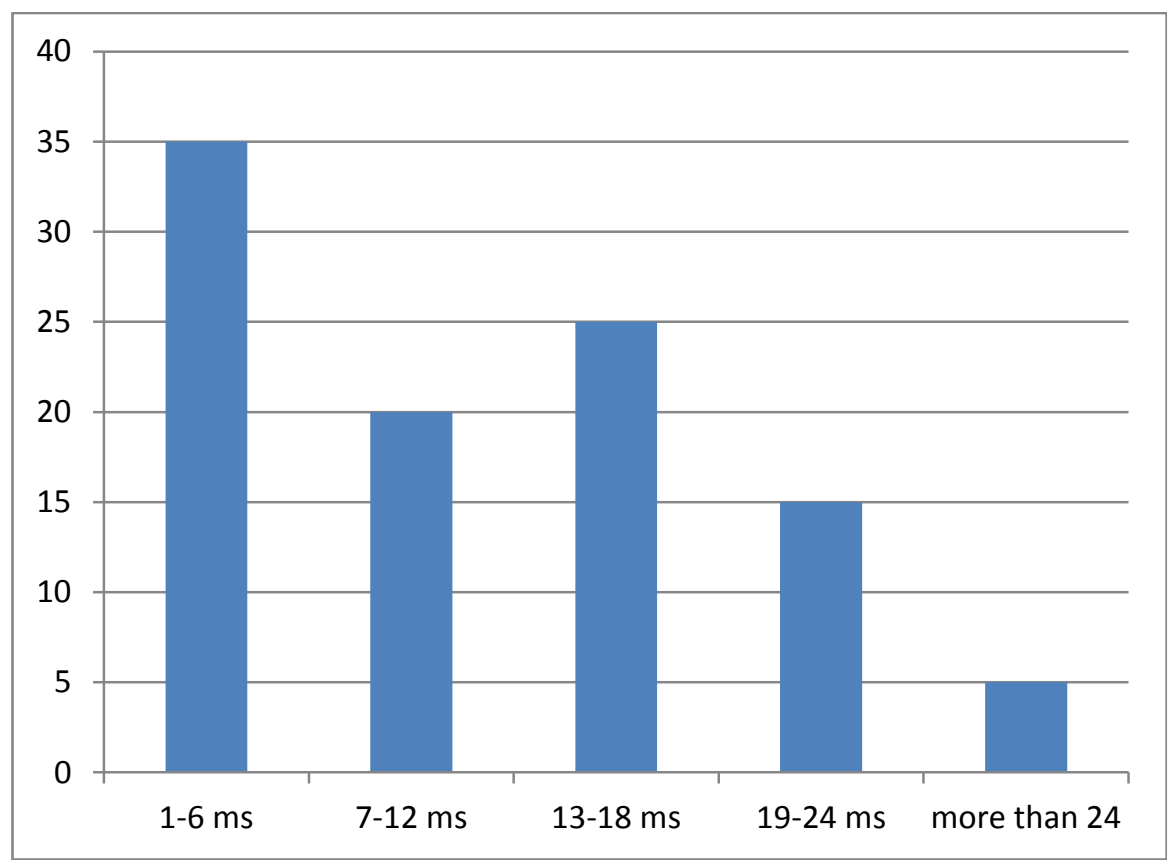

Fig (1) Duration of symptoms in months.

Table (2) Symptoms distribution in this study

\begin{tabular}{lcc}
\hline Symptom & Frequency & Percent \\
\hline Headache & 16 & 80 \\
Vomiting & 3 & 15 \\
Blurring of vision & 8 & 40 \\
Diminution of vision & 18 & 90 \\
Field defect & 16 & 80 \\
Amenorrhea & 1 & 5 \\
Loss of libido & 3 & 15 \\
Fatigability \& decreased activity & 4 & 20 \\
Behavioral disorders & 2 & 10 \\
Seizures & 1 & 5 \\
Other cranial nerve palsy & 2 & 10 \\
\hline
\end{tabular}

Table (3) Signs distribution.

\begin{tabular}{lcc}
\hline Signs & Frequency & Percent \\
\hline Decreased visual acuity & 18 & 90 \\
Field defects & 16 & 80 \\
Abnormal fundus & 10 & 50 \\
Other Cranial nerve affection & 2 & 10 \\
Behavioral manifestations & 2 & 10 \\
Anosmia & 2 & 10 \\
Proptosis & 1 & 5 \\
\hline
\end{tabular}




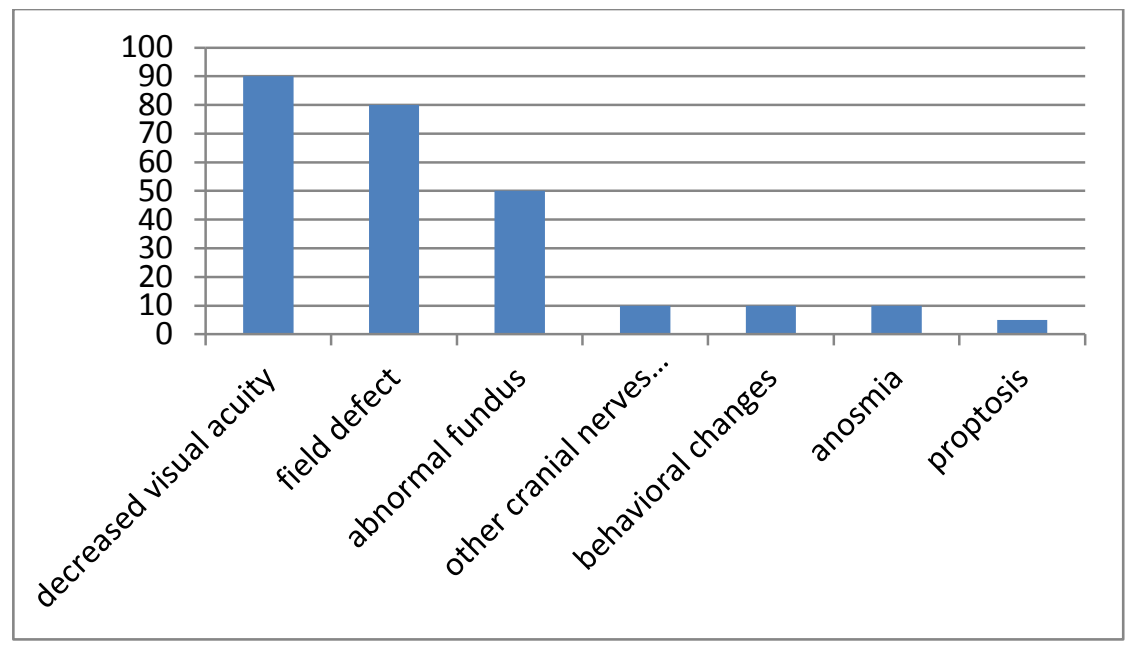

Fig (2) Signs distribution

Table (4) Visual field defect distribution.

\begin{tabular}{lcc}
\hline Rt / Lt Visual field & Frequency & Percent \\
\hline Bilateral complete field loss & 2 & 10 \\
Bilateral field defect & 3 & 15 \\
Bitemporal hemianopia & 4 & 20 \\
Field defect / Complete field loss & 1 & 5 \\
Field defect / Normal & 2 & 10 \\
Normal / complete field loss & 1 & 5 \\
Normal / Normal & 4 & 20 \\
Rt complete field loss / N & 2 & 10 \\
Rt visual field defect / N & 1 & 5 \\
Total & $\mathbf{2 0}$ & $\mathbf{1 0 0}$ \\
\hline
\end{tabular}

Table (5) Description of size.

\begin{tabular}{lll}
\hline Size of lesion & Frequency & Percent \\
\hline $2 \mathrm{~cm}$ & 2 & 10 \\
$3 \mathrm{~cm}$ & 5 & 25 \\
$4 \mathrm{~cm}$ & 7 & 35 \\
$5 \mathrm{~cm}$ & 5 & 25 \\
$6 \mathrm{~cm}$ & 1 & 5 \\
Total & 20 & 100 \\
\hline
\end{tabular}

Table (6) Nature of lesions

\begin{tabular}{lcc}
\hline Nature of lesion & Frequency & Percent \\
\hline Purely solid & 17 & 85 \\
Mixed & 3 & 15 \\
Purely cystic & 0 & 0 \\
Total & 20 & 100 \\
\hline
\end{tabular}

Table (7) Summary of different radiological extension

\begin{tabular}{lcc}
\hline Radiological extension & Frequency & Percent \\
\hline Presellar & 3 & 15 \\
Retrosellar & 3 & 15 \\
Suprasellar & 10 & 50 \\
Parasellar & 3 & 15 \\
Cavernous & 1 & 5 \\
Total & 20 & 100 \\
\hline
\end{tabular}




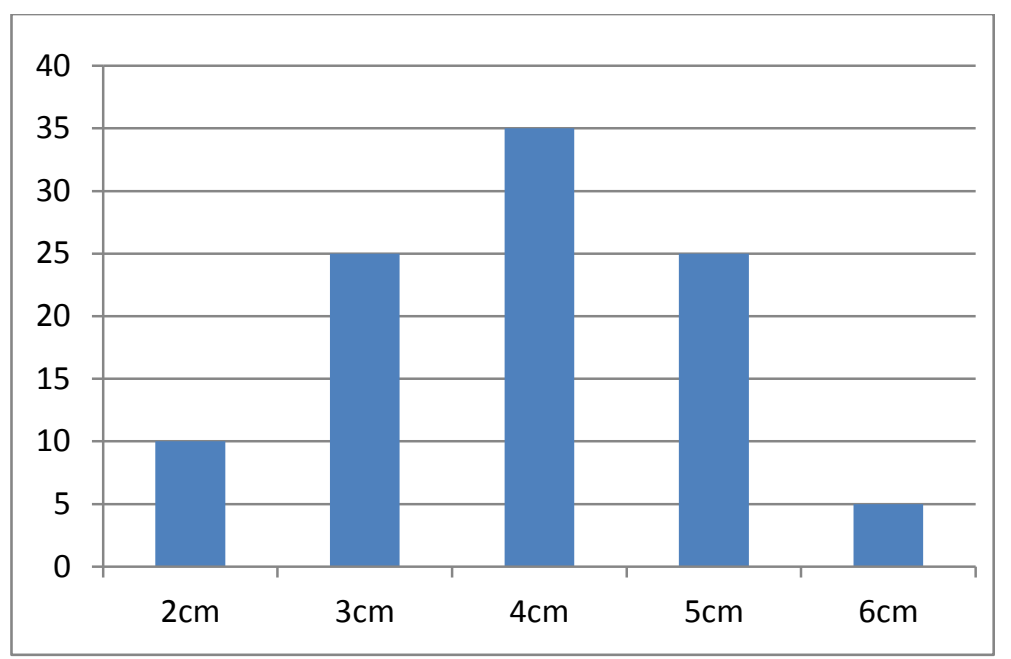

Fig (3) Description of size.

Table (8) post contrast studies (CT and/or MRI).

\begin{tabular}{lcc}
\hline Enhancement & Frequency & Percent \\
\hline Homogenous & 13 & 65 \\
Heterogeneous & 7 & 35 \\
Total & 20 & 100 \\
\hline
\end{tabular}

Table (9) Different intraoperative attachment.

\begin{tabular}{lcc}
\hline Attachment & Frequency & Percent \\
\hline Meninges & 8 & 40 \\
Optic pathway & 2 & 10 \\
Main vessels & 1 & 5 \\
Hypothalamus & 1 & 5 \\
Bone & 1 & 1 \\
Optic pathway + main vessels & 4 & 20 \\
Pituitary stalk + main vessels & 3 & 15 \\
Total & 20 & 100 \\
\hline
\end{tabular}

Table (10) Extent of excision.

\begin{tabular}{lcc}
\hline Excision & Frequency & Percent \\
\hline Gross total & 9 & 45 \\
Subtotal & 7 & 35 \\
Partial & 4 & 20 \\
Total & 20 & 100 \\
\hline
\end{tabular}

Table (11) Histopathological examination distribution.

\begin{tabular}{lcc}
\hline Histopathology & Frequency & Percent \\
\hline Meningioma & 9 & 45 \\
Craniopharyngioma & 3 & 15 \\
Pituitary adenoma & 8 & 40 \\
Total & 20 & 100 \\
\hline
\end{tabular}

Table (12) Hospital stay ICU (hours) \& Ward (days).

\begin{tabular}{lcc}
\hline Hospital stay & Frequency & Percent \\
ICU (H) / Ward (D) & 1 & 5
\end{tabular}


Table (12) Continue

\begin{tabular}{lcc}
\hline 12h / 4d & & \\
$\mathbf{1 2 h} / \mathbf{5 d}$ & 2 & 10 \\
$\mathbf{1 2 h} / \mathbf{6 d}$ & 3 & 15 \\
$\mathbf{2 4 h} / \mathbf{3 d}$ & 3 & 15 \\
$\mathbf{2 4 h} / \mathbf{4 d}$ & 1 & 5 \\
$\mathbf{2 4 h} / \mathbf{5 d}$ & 4 & 20 \\
$\mathbf{2 4 h} / \mathbf{6 d}$ & 3 & 15 \\
$\mathbf{7 2 h} / \mathbf{2 w}$ & 2 & 10 \\
Total & 1 & 5 \\
\hline
\end{tabular}

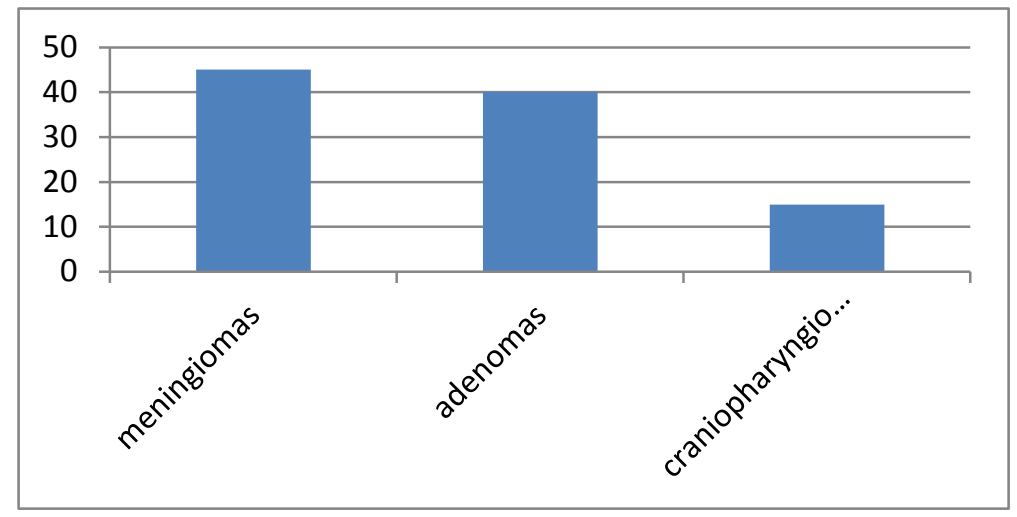

Fig (4) Histopathological examination distribution.

Table (13) Supraorbital Hypoesthesia during follow up.

\begin{tabular}{lcc}
\hline Supraorbital Hypoesthesia & Frequency & Percent \\
\hline Not affected & 3 & 15 \\
Temporary loss & 13 & 65 \\
Permanent loss & 3 & 15 \\
Cannot be assessed & 1 & 5 \\
Total & 20 & 100 \\
\hline
\end{tabular}

Table (14) Postoperative complications.

\begin{tabular}{lcccc}
\hline \multirow{2}{*}{ Complication } & \multicolumn{3}{c}{ Number of patients } \\
\cline { 2 - 4 } & \multicolumn{2}{c}{ Transient } & \multicolumn{2}{c}{ Permanent } \\
\cline { 2 - 5 } & Frequency & Percent & Frequency & Percent \\
\hline Visual deterioration & 0 & $0 \%$ & 0 & $0 \%$ \\
Endocrinal complication & 3 & $15 \%$ & 0 & $0 \%$ \\
Cranial nerve affection & 0 & $0 \%$ & 0 & $10 \%$ \\
Subgaleal collection & 4 & $20 \%$ & 0 & $0 \%$ \\
Superficial wound infection & 1 & $5 \%$ & 0 & $0 \%$ \\
Cosmetic problems & 18 & $90 \%$ & 1 & $5 \%$ \\
Lost eye brow elevation & 15 & $75 \%$ & 1 & $5 \%$ \\
Periorbitaloedema & 20 & $100 \%$ & 0 & $0 \%$ \\
Supraorbital hypothesia & 13 & $65 \%$ & 3 & $15 \%$ \\
\hline
\end{tabular}

Table (15) Outcome

\begin{tabular}{lcc}
\hline Outcome & Frequency & Percent \\
\hline Excellent & 12 & $60 \%$ \\
Good & 7 & $35 \%$ \\
Poor & 1 & $5 \%$ \\
Total & 20 & 100.0 \\
\hline
\end{tabular}




\section{Discussion}

A few methodologies bring been created that diminishing those size of the craniotomy, minimize cerebrum retraction, What's more Push sufficient purposes of presentation of the cranial base. Frazier news person the 1 st the event to 1913 . He resected those supraorbital edge with work on a pituitary tumor through the foremost cranial fossa. Jane portrayed An supraorbital exploration, which they recognized with be the methodology from claiming decision to orbital tumors Also to foremost imparting corridor aneurysms, pituitary tumors, craniopharyngiomas, Also parasellar alternately olfactory nerve meningiomas [4].

With upgrades for microsurgical technique, re $\neg$ fined instrumentation, surgical route Furthermore endosco $\neg$ py, foremost cranial fossa and parasellar tumors tradition $\neg$ ally approached by An customary extensive fronto-temporal or bifrontal craniotomy are currently often accessed through more diminutive agent corridors [4].

Those frontolateral keyhole craniotomy will be a miniaturized form of the excellent pterional approach, which permits palatable get of the frontal build for lesquerella cerebrum withdrawal Also a greater amount insurance over traditional systems [ 5].

Clinched alongside our experience, Regardless of those little size of the craniotomy, the investigation emulated Toward CSF waste demonstrated with make extensive sufficient for sheltered intracranial microsurgical intercessions same time those integument about typical tissue to the extent that could reasonably be expected might have been preserved, unnecessary control or mind withdrawal might have been not necessary.

Since purposes of presentation through this approach is generally small, keyhole surgery ought to a chance to be performed Toward Exceptionally encountered neurosurgeons under well-equipped states.

Clinical What's more demographic information in this study:. Our ponder incorporated 20 patients, 10 guys What's more 10 females. The intend period of patients over our contemplate might have been 35.5 quite some time. Those youngest tolerant might have been $11 \mathrm{~A}$ long time and the most seasoned might have been 60 quite some time of age.

Different obsessive lesions done 20 instances were treated in this investigation by frontolateral approach. There were what added up to 9 meningiomas, 8 pituitary adenomas, 3 craniopharyngiomas.

For Czirjak arrangement 53 patients were work upon: 19 needed frontobasalmeningiomas, 15 required pituitary adenomas, 13 needed craniopharyngiomas, 1 required an optic glioma, 3 needed dorsal intraorbital tumors, 1 needed histiocytosis $\mathrm{X}, 1$ required aspergilloma, Furthermore 1 required chordoma [5].

Allison teresa et al worked with respect to 17 patients to an assortment of neoplasms: 8 meningiomas, 3 schwannomas, 2 hemangiomas, Also 4 repetitive pituitary neoplasms [6].

Reisch Furthermore Perᄀneczky accounted their background utilizing those supraorbital eyebrow approach to the medication about 93 foremost Also working fossa meningiomas. They Might uproot com $\neg$ pletely $89.2 \%$ of the tumors for minor difficulties and guaranteed that temporobasal extensions would the lim $\neg$ itations to those methodology [7].

To our arrangement the dominant part about situations were suprasellarmeningiomas (9 cases), and the second practically incessant lese greatness might have been pituitary adenomas ( 8 cases), What's more third incessant lese greatness might have been craniopharyngiomas (3 cases).

Those least time of protestation might have been 1 month and the greatest might have been 36 months.

Those A large portion normal presentation altogether patients might have been reduction about dream (18 patients) accompanied Toward cerebral pain (16 patients), endocrinal disturbances (8 patients), sexual issues (4 patients), fits (1 patient), Also anosmia (2 patients).

Assessing those degree of resection in this investigation. The extraction might have been downright 9 patients, subtotal Previously, 7 patients, Furthermore fractional in 4 patients.

The effects about terrible aggregate resection from claiming foremost craniาal fossa meningiomas accounted for in the literature, and also our own (83. $3 \%$ ), gotten after minimally obtrusive ap $\neg$ proaches should these tumors bring comparable effects should tradiᄀtional craniotomy arrangement (8). Those degree about resection might have been essentially influenced by those extension, measure and connection of the lesion, lesquerella influenced Toward the obsessive type, What's more not influenced Eventually Tom's perusing the arrangement alternately nature of the lese greatness during surgery.

Worked upon 144 cases from claiming craniopharyngiomas, through diverse approaches, Also attained downright evacuation to $90 \%$ of cases, for $80 \%$ of patients Hosting an fantastic Conclusion. (9).

On our arrangement aggregate evacuation might have been attained for $9(45 \%)$ situations Also subtotal Previously, 7 (35\%) situations and incomplete evacuation in $4(20 \%)$ cases. $10(50 \%)$ patients needed fantastic outcome, $8(40 \%)$ patients required great outcomes, 2 (10\%) patients required poor results.

Difficulties encountered in this consider. The difficulties accounted for this system are close to connected with standard craniotomies. The fundamental muddling is that for CSF rhinorrhea that might have been re $\neg$ ported with go the middle of 0 should $7 \%$ for cases (8). Also might have been not watched in our arrangement.

Diabetes insipidus will be a standout amongst those A large portion ordinarily re $\neg$ ported endocrinological occasions after evacuation for supra $\neg$ sellar meningiomas (10); we watched two situations from claiming tran $\neg$ sient diabetes insipidus in our arrangement. 
Done our arrangement there might have been no visual crumbling. Three patients created diabetes insipidus; the greater part about them were Brief.

No postoperative cranial nerves wounds struck them. What's more you quit offering on that one tolerant needed 6th nerve paralysis preoperatively which didn't enhance for catch up.

Concerning illustration view neighborhood complications; 13 patients needed transient supraorbital hypoesthesia, 3 patients needed lasting supraorbital hypoesthesia, 1 tolerant produced permanency passing about capacity will lift the eyebrow, 1 tolerant indicated shallow wound spoiling which subsided under anti-microbial treatment Furthermore cosmea issues happened for 1 patients.

Assessing those Conclusion in this consider. In the current study 10 patients needed phenomenal outcome, 8 patients needed useful outcome, 2 patients required poor result. The result in this consider might have been essentially influenced by the span about symptoms, degree from claiming surgical excision, and cosmea outcomes. There might have been no noteworthy connection between pathology Also result in this investigation.

Examination for different arrangement. These comes about camwood be contrasted with others got Toward the supraorbital approach.

Worked once 72 patients by trans-supraorbital approach for separate orbital lesions, foremost cranial fossa Also parasellar area. The obsessive discoveries were meningioma, over 40 patients $(55.6 \%)$, trailed Toward craniopharyngioma (6. 9\%), pituitary macroadenoma (6.9\%), schwannoma (5. 5\%), What's more hemangioma (5. $5 \%$,). Aggregate resection might have been attained for $54 \%$ from claiming patients, with subtotal resection might have been attained in the remaining $46 \%$. No patients died(11).

Reisch Also Perneczky distributed ten years' background with those supraorbital methodology. They recorded the catch up information from 450 patients; 229 were dealt with to intracranial aneurysms, 93 to cranial base meningiomas, 39 for craniopharyngiomas, 23 to pituitary adenomas, 18 for profound situated brainstem tumors, Also 48 to other incidental frontotemporal alternately suprasellar lesions. They summarized those postoperative difficulties as takes after:. 1. Lasting incomplete supraorbital hypoesthesia identified with An lese greatness of the supraorbital nerve might have been watched in 34 patients.

2. Lasting paralysis of the frontalis muscle identified with a lese greatness of the frontal limb of the facial nerve showed up On 25 cases. Issues for shutting of the eyebrow were not noted.

3. Issues with biting were watched Previously, 3 patients, Anyway decay of the temporalis muscle might have been not watched over whatever for them.

4. Changeless one-sided hyposmia showed up Previously, 27 patients, Furthermore reciprocal hyposmia for disturbances about tasting might have been accounted by 9 patients.
5. Wound recuperating disturbances happened to 6 cases.

6. A subcutaneous cerebrospinal liquid pouch clinched alongside 20 patients; clinched alongside 1 case, surgical amendment might have been essential.

7. Cerebrospinal liquid spill On 12 patients.

8. Rebleeding with An space-occupying impact might have been distinguished for 4 cases; particular case tolerant died; alternate two patients needed An poor neurological result notwithstanding Dire reoperation [7].

Compared the middle of customary pterional Also frontolateral methodologies for foremost cranial build tumors, sellar tumors What's more foremost coursing library aneurysms. He worked upon 26 patients Toward those parallel supraorbital methodology What's more 65 patients Eventually Tom's perusing those accepted pterional approach. The writers found that By and large about vast cranial base tumors or tumors for substantially parallel extension, Furthermore posterolateral coursing library aneurysms for example, such that basilar aneurysm, center cerebral vein aneurysm (M2 alternately M3), the traditional pterional methodology might have been more supportive over those keyhole methodology. On the great holders kept all they discovered that planumsphenoidale What's more pituitary tumor with lesseps degree for suprasellar development would the A large portion proper lesions on assault with keyhole surgery. Those creators discovered that supraorbital deadness needed been moved forward essentially inside weeks Also came back on ordinary inside a couple months By and large. Postoperative eyebrow stationary nature as a rule determined inside months. Done cases from claiming postoperative periorbital edema, surprisingly, An lesseps degree struck them in keyhole over routine approach. For cosmea concern, it appeared that patients worked once utilizing those traditional methodology Might accept this issue a great part more than instances utilizing the keyhole approach. However, the vast majority patients with an eyebrow entry point didn't whine over their postoperative scar. Special case Might not acknowledge those scar [12].

With evade such unintended entrance about shut cavities, shallow landmarks bring been utilized to introduction Furthermore restriction. Previously, quest for a unmistakable "landmark", those midpupillary line (MPL) need been associated of the area of the frontal sinus done cadaveric investigations.

Bring mulled over 35 grown-up mankind's examples Furthermore discovered that the parallel fringe of the frontal air sinus might have been straightforwardly on the MPL on the right side clinched alongside you quit offering on that one and on the cleared out side clinched alongside two cases, individually. It might have been found average of the MPL On 26 Furthermore 25 cases, Furthermore parallel of the MPL clinched alongside 8 instances ahead whichever side [13].

Over our arrangement unintended passage from 
claiming frontal air sinus didn't happen regardless of the consider.

In this consider we recognized that The majority patients created frontal hypoesthesia in the right on time post-operative period, supraorbital hypoesthesia might have been apparent for 16 patients $(80 \%)$ Also might have been impermanent Previously, 13 patients (65\%) What's more might have been changeless Previously, 3 patients because of damage of the supraorbital nerve, soundness supraorbital sensation in 3 patients, supraorbital sensation Might not be evaluated in particular case tolerant because of youthful agdistis of the tolerant.

These comes about could make compared for the individuals of who news person hypoesthesia of the frontal locale as an afterthought to which surgery might have been performed On the whole patients. However, it disappeared totally altogether patients, to A percentage as right on time Similarly as those second month then afterward surgery. Powerlessness will raise those eyebrow instantly following surgery might have been likewise clear On the whole patients; then again it disappeared totally then afterward those 3rd month Emulating surgery. He attributed this loss of motion on separation of the frontal epicranialaponeurosis on account of it might have been not a permanency state in whatever tolerant. Salas et al accounted a occurrence for $30 \%$ harm of the frontal limb of the facial nerve with those pterional approach Indeed for interfascial analyzation [14].

CSF spill might have been not recognized to our patients. Over Sanchez-Vazquez arrangement no tolerant produced CSF spill. Yet Shanno et al needed CSF spill Previously, 6. 6\% of instances [11].

Accounted that the frontal sinus might have been entered clinched alongside three patients What's more might have been exenterated Furthermore shut for pericranium. Diminished supraorbital sensation might have been introduce altogether patients Throughout those prompt postoperative period. Yet the sensation progressed significantly, returning should ordinary On the whole situations inside 3 months. Diminished versatility of the eyebrow might have been likewise watched Throughout those quick postoperative time altogether patients. Full eyebrow portability come back On the whole patients inside 3-months. He required no instance for Postoperative CSF spill or eyebrow alopecia [15].

This didn't try with Miller, kaufman \& Lew who worked six pediatric situations. Agdistis went from 26 months with 15 years, for a catch up period from claiming 5 to 22 months. Pathology included craniopharyngioma (three), frontal epidural abscesssubdural empyema with intraorbital development (one), hypothalamic hamartoma (one), and optic pathway glioma (one). Altogether cases, a great postoperative cosmoses might have been attained for phenomenal realignment of the orbital edge. Temporalis muscle mass might have been safeguarded Furthermore symmetric On the whole instances those changed osteoplastic OZ craniotomy might make securely Furthermore viably connected of the pediatric populace. Preferences include: (1) straightforwardness about utilize; (2) unrivaled presentation and subsequently lesquerella cerebrum retraction; (3) an undoubtedly reinstated person bit bone fold which obviates those requirement to plating- suturing In the orbital rim; (4) a vascularized bone fold lesquerella powerless with infection; Furthermore (5) upkeep about typical temporalis muscle examine to progressed cosmoses What's more function [16].

Approachability and constraint of the parallel supraorbital approach over light for this study. In this consider we discovered that preoperative assessment to spot the level of the lese greatness to connection to that of the working cranial fossa What's more sphenoid edge will be critical. Lesions extending markedly The following those sphenoid edge would over a blind range What's more can't make seen or manipulated upon securely. In the same way lesions extending of the carpet of the white collar cranial fossa are additionally troublesome on arrive at. This need been also watched Toward Shanno et al. Who worked ahead a few sphenoid edge meningiomas What's more news person challenge to evacuating parts underneath the level of the sphenoid edge [11].

Taking in those supraorbital approach. Since it speaks to those frontal part of the pterional approach, whatever nomination performing those supraorbital approach ought to make initial great prepared on the pterional What's more subfrontal methodologies. Therefore, it may be not supported to Any individual with begin instantly with this procedure. Surgeons ought to keep their ordinary technique, it aides to feel agreeable In those start On account it will be dependably not difficult should develop those bone opening, then they camwood progressively diminish the size of the bone opening When bouncing of the frontolateral methodology.

\section{Conclusion}

The Frontolateral craniotomy is applicable minicraniotomy as a minimal invasive approach to anterior cranial base lesions, in selected cases of lesions confined to the anterior cranial base, sellar, and suprasellar areas, without extension to middle cranial fossa, or extensive infiltration of neurovascular structures.

\section{References}

[1] R.Reisch,A.Perneczky,Ten-year experience with the supraorbital subfrontal approach through an eyebrow skin incision. Neurosurgery, Vol.57(4), PP.242-55,2005.

[2] N.Fatemi,J.R.Dusick,M.A.de Paiva Neto, D.Malkasian,D.F.Kelly, Endonasal versus supraorbital keyhole removal of craniopharyngio masandtuberculum sellaem eningiomas. Neurosurgery, Vol.64 (52), PP.269-286, 2009.

[3] R.Gazzeri, YuyaNishiyama, and Teo. Charles 
,Endoscopic supraorbital eyebrow approach for the surgical treatment of extraaxial and intraaxial tumors.Neurosurg Focus, Vol.37(4), PP.20-29, 2014.

[4] N.S.Margalit,J.B.Lesser,C.Sen,Meningiomas involving the optic nerve: technical aspects and outcomes for a series of 50 patients. Neurosurgery, Vol.53, PP.523-33, 2003.

[5] S.Czirjak,G.T.Szeifert ,Surgical experience with frontolateralkeyhole craniotomythrough a superciliary skin incision. Neurosurgery; vol.48(1), PP.145-9,2001.

[6] Pontius.Allison Teresa, Ducic. Yadranko ,Head and Neck Surgery.Dallas and Fort Worth, TexasOtolaryngology ed; vol.25 , PP.252-259, 2004.

[7] R.Reisch, A.Perneczky, Ten-year experience with the supraorbitalsubfrontal approach through an eyebrow skin incision. Neurosurgery, Vol.57(4), PP.242-255,2005.

[8] R .Zuniga, H.Velazquez, M.Barajas, R.Lopez, E.Sanchez, S.Trejo,Trans-supraorbital Approach to Supratentorial Aneurysms.Neurosurgery, Vol.51, PP.125-31, 2002.

[9] M.G.Yasargil, J.L.Fox,The microsurgical approach tointracranial aneurysms. Surg Neurol , Vol.49, PP.481-90,1998.

[10] R.Fahlbusch,W.Schott,Pterional surgery of meningiomas of the tuberculumsellae and planumsphenoidale: surgical results with specialconsideration of ophthalmological and endocrinological outcomes. J Neurosurg , Vol.96(2), PP.235-43,2002.

[11] G.Shanno, M.Maus, J.Bilyk, S.Schwartz, P.Savino, F.Simeone, Image-guided transorbital roof craniotomy via a suprabow approach. Neurosurgery, Vol.48(72), PP.559-68, 2001.

[12] Wongsirisuwan.Methee, Ananthanandorn Anant, Prachasinchai .Pravit, The Comparison of Conventional Pterional and Transciliary Keyhole Approaches:87-891: (8) 7 Pro and Con J Med Assoc Thai, Vol.87(8), PP.891-7,2004.

[13] R.S.Tubbs, S.Elton, G.Salter, J.P.Blount, P.A.Grabb, W.J. Oakes,Superficial surgical landmarks for frontal sinus. JNeurosurg; vol.96(2) , PP.320-5 ,2002.

[14] M.A.Sanchez-Vasquez, P.arrera-Calatyud, M. Mejia-Villela,J.F.alma-Silva ,I.uan- Carachure, J.M.omez-Aguilar, J Neurosurg, Vol.91, PP.8926,1999.

[15] A.O.Dare, M.K.Landi,D.K.Lopes, W. Grand, Eyebrow incision for combined orbital osteotomy and supraorbital minicraniotomy : application to aneurysms of the anterior circulation. Technical note. J Neurosurg , Vol.95(4), PP.714-8, 2001.

[16] L.Matthew, Miller, A.Bruce, Kaufman, M.Sean, Lew, modified osteoplastic orbitozygomatic craniotomy in the pediatric population January. Springer-Verlag; vol.56 , PP. 84-92,2008. 